\title{
A Dependent Insurance Risk Model with Surrender and Investment under the Thinning Process
}

\author{
Wenguang $\mathrm{Yu}^{1}$ and Yujuan Huang ${ }^{2}$ \\ ${ }^{1}$ School of Insurance, Shandong University of Finance and Economics, Jinan 250014, China \\ ${ }^{2}$ School of Science, Shandong Jiaotong University, Jinan 250023, China \\ Correspondence should be addressed to Yujuan Huang; yujuanh518@163.com
}

Received 26 August 2015; Accepted 17 September 2015

Academic Editor: Xinguang Zhang

Copyright (c) 2015 W. Yu and Y. Huang. This is an open access article distributed under the Creative Commons Attribution License, which permits unrestricted use, distribution, and reproduction in any medium, provided the original work is properly cited.

\begin{abstract}
A dependent insurance risk model with surrender and investment under the thinning process is discussed, where the arrival of the policies follows a compound Poisson-Geometric process, and the occurrences of the claim and surrender happen as the $p$ thinning process and the $q$-thinning process of the arrival process, respectively. By the martingale theory, the properties of the surplus process, adjustment coefficient equation, the upper bound of ruin probability, and explicit expression of ruin probability are obtained. Moreover, we also get the Laplace transformation, the expectation, and the variance of the time when the surplus reaches a given level for the first time. Finally, various trends of the upper bound of ruin probability and the expectation and the variance of the time when the surplus reaches a given level for the first time are simulated analytically along with changing the investment size, investment interest rates, claim rate, and surrender rate.
\end{abstract}

\section{Introduction}

In the classical ruin theory, compound Poisson risk model,

$$
U(t)=u+c t-\sum_{i=1}^{N(t)} X_{i} \text { for } t \geq 0
$$

is the main research object $[1,2]$, where $u \geq 0$ is the initial reserve, $c$ is the premium rate, and $\{N(t), t \geq 0\}$ is a Poisson process with intensity $\lambda>0$, representing the number of claims up to time $t$. The individual claim sizes $X_{1}, X_{2}, \ldots$, independent of $\{N(t), t \geq 0\}$, are i.i.d. positive random variables with distribution function $F(x)$ and density function $f(x)$ with mean $\mu$. In the model, the premium income process is a linear function of time; it does not matter to claim. But in actual life, the arrival of policy of insurance company is usually associated with occurrence of claim; for example, the more the number of policies sold, the more the number of claims happened. Therefore, many studies in literature discuss the dependent relationship among the premium income, interclaim arrivals, and the claim size. See, for example, Liu et al. [3] considering a Markov-dependent risk model with a constant dividend barrier. Shi et al. [4] explore methods that allow for the correlation among frequency and severity components for microlevel insurance data. Jiang et al. [5] investigate some uniform asymptotic estimates for finitetime ruin probabilities when the claim size vector and its interarrival time are subject to certain general dependence structure. Zhang and Yang [6], Shi et al. [7], and Zou et al. [8] consider a compound Poisson risk model and a dependence structure of the claim size and interclaim time modeled by a Farlie-Gumbel-Morgenstern copula.

The above papers always assume the claim number follows a Poisson distribution, but in fact the claim number does not fully comply with the rule of Poisson distribution and its variance is often greater than the mean. Except the natural environment, an important reason for this phenomenon is that insurance companies have adopted risk aversion mechanism, such as franchise system and no-claim discount system [9]. This makes the policy holder weighs the interests which may not claim for compensation in the event of an accident; it will cause the claim number to be less than the number of accidents. In addition, on the one hand, the insurance company will have huge funds and various kinds of reserves in the operation process, which formed the huge amount of available funds. On the other hand, in order to 
protect the interests of the insured, the insurance company must use the fund rationally and effectively. In fact, the insurance industry is very active in the financial markets. In the financial markets of western developed countries, the total amount of funds provided by the insurance industry is close to commercial banks. So considering the risk model with investment income has greater practical value and realistic significance [10-12].

In view of the above problems, this paper will promote the premium income process of insurance companies to follow the compound Poisson-Geometric process [13-15], while the counting processes of claim and surrender are the $p$-thinning process and the $q$-thinning process of premium income process and further consideration of the investment interest rate. For the new improved model, we study the properties of surplus process, adjustment coefficient equation, ruin probability, and the expectation and variance of the first time to reach a given level. Finally, numerical analysis is also given.

The contents of this paper are organized as follows: Section 2 introduces the risk model. In Section 3, we give the main results of the paper. Finally, we provide the numerical examples in Section 4.

\section{The Risk Model}

Definition 1. Let $u \geq 0$ and $(\Omega, F, P)$ be a probability space; $t \geq$ 0 ; then, the surplus process with initial surplus $u$ is defined as follows:

$$
\begin{aligned}
U(t)= & u+A \alpha t+\sum_{k=1}^{N(t)} X_{k}-\sum_{k=1}^{N(t, p)} Y_{k}-\sum_{k=1}^{N(t, q)} Z_{k} \\
& +\beta W(t),
\end{aligned}
$$

where $u$ represents the initial capital and $A(A<u)$ represents the investment capital, which is based on the size of initial capital, premium income per unit of time, and the predicted claim sizes. $\alpha$ represents the investment income per unit of time. $\{N(t) ; t \geq 0\}$ is a Poisson-Geometric process with parameters $\lambda(\lambda>0)$ and $\rho(0<\rho<1)$ denoting the number of premiums up to time $t$; namely, $N(t) \sim P G(\lambda t, \rho)$. $\left\{X_{k} \geq 0 ; k \geq 1\right\}$ is a sequence of i.i.d. random variables representing the amount of the $k$ th premium and $E\left[X_{k}\right]=$ $\mu_{X} \operatorname{Var}\left[X_{k}\right]=\sigma_{X}^{2} \cdot\{N(t, p) ; t \geq 0\}$ is the $p$-thinning process of $\{N(t) ; t \geq 0\}$ denoting the number of claims up to time $t$; namely, $\{N(t, p) ; t \geq 0\} \sim P G(\lambda p t, \rho)$. The individual claims sizes $\left\{Y_{k} \geq 0 ; k \geq 1\right\}$ are a sequence of i.i.d. random variables and $E\left[Y_{k}\right]=\mu_{Y} \operatorname{Var}\left[Y_{k}\right]=\sigma_{Y}^{2} \cdot\{N(t, q) ; t \geq 0\}$ is the $q-$ thinning process of $\{N(t) ; t \geq 0\}$ denoting the number of surrenders up to time $t$; namely, $\{N(t, q) ; t \geq 0\} \sim P G(\lambda q t, \rho)$ and $0<q<1$ and $0<p+q<1$. The sequence of i.i.d. random variables $\left\{Z_{k} \geq 0 ; k \geq 1\right\}$ represents the amount of the $k$ th payment of insurance policy and $E\left[Z_{k}\right]=\mu_{Z}, \operatorname{Var}\left[Z_{k}\right]=\sigma_{Z}^{2}$, and $\mu_{Z}<\mu_{X} .\{W(t) ; t \geq 0\}$ is a standard Brownian motion denoting the uncertain benefits and payments of insurance companies. $\beta>0$ is a constant, representing the diffusion volatility parameter. In addition, we suppose that $\left\{X_{k}, k \geq\right.$ $1\},\left\{Y_{k}, k \geq 1\right\},\left\{Z_{k}, k \geq 1\right\},\{W(t), t \geq 0\}$, and $\{N(t) ; t \geq 0\}$ are mutually independent. From the theory of point process,
$\{N(t, p) ; t \geq 0\}$ and $\{N(t, q) ; t \geq 0\}$ are also mutually independent.

Let $S(t)=A \alpha t+\sum_{k=1}^{N(t)} X_{k}-\sum_{k=1}^{N(t, p)} Y_{k}-\sum_{k=1}^{N(t, q)} Z_{k}+\beta W(t)$ be profits process. In order to ensure the insurance company's steady business, we assume $E[S(t)]>0$, and the relative security loading factor $\theta$ is defined as follows:

$$
\theta=\frac{\lambda \mu_{X}+A \alpha(1-\rho)}{\lambda p \mu_{Y}+\lambda q \mu_{Z}}-1>0 .
$$

\section{Main Results}

Lemma 2. The profits process $\{S(t), t \geq 0\}$ has the following properties:

(i) $\{S(t), t \geq 0\}$ has stationary and independent increments.

(ii) $E[S(t)]=\left(A \alpha+\lambda \mu_{X} /(1-\rho)-\lambda p \mu_{Y} /(1-\rho)-\lambda q \mu_{Z} /(1-\right.$ $\rho)) t$.

Lemma 3. For the profits process $\{S(t), t \geq 0\}$, when $E[S(t)] \geq$ 0 , one has the following:

$$
\lim _{t \rightarrow \infty} U(t)=\infty, \quad \text { a.s. }
$$

Lemma 4. For the profits process $\{S(t), t \geq 0\}$, suppose $E\left[e^{-r S(t)}\right]<0$ for some $r>0$; then, there is a function $g(r)$ such that

$$
E\left[e^{-r S(t)}\right]=e^{t g(r)}
$$

Proof. Consider

$$
\begin{aligned}
& E[\exp (-r S(t))]=E[\exp (-r A \alpha t)] \\
& \cdot E\left[\exp \left(-r \sum_{K=1}^{N(t)} X_{k}\right)\right] \cdot E\left[\exp \left(r \sum_{K=1}^{N(t, p)} Y_{k}\right)\right] \\
& \cdot E\left[\exp \left(r \sum_{K=1}^{N(t, q)} Z_{k}\right)\right] \cdot E[\exp (-r \beta W(t))] \\
& =\exp \left\{t \left[-r A \alpha+\frac{\lambda\left(M_{X}(-r)-1\right)}{1-\rho M_{X}(-r)}\right.\right. \\
& \left.\left.+\frac{\lambda p\left(M_{Y}(r)-1\right)}{1-\rho M_{Y}(r)}+\frac{\lambda q\left(M_{Z}(r)-1\right)}{1-\rho M_{Z}(r)}+\frac{1}{2} \beta^{2} r^{2}\right]\right\} .
\end{aligned}
$$

Let

$$
\begin{aligned}
g(r)= & -r A \alpha+\frac{\lambda\left(M_{X}(-r)-1\right)}{1-\rho M_{X}(-r)}+\frac{\lambda p\left(M_{Y}(r)-1\right)}{1-\rho M_{Y}(r)} \\
& +\frac{\lambda q\left(M_{Z}(r)-1\right)}{1-\rho M_{Z}(r)}+\frac{1}{2} \beta^{2} r^{2},
\end{aligned}
$$

where $M_{X}(r)=E\left[e^{r X}\right]$ is the moment generating function of $X$. Similarly, we can define $M_{Y}(r)$ and $M_{Z}(r)$. 


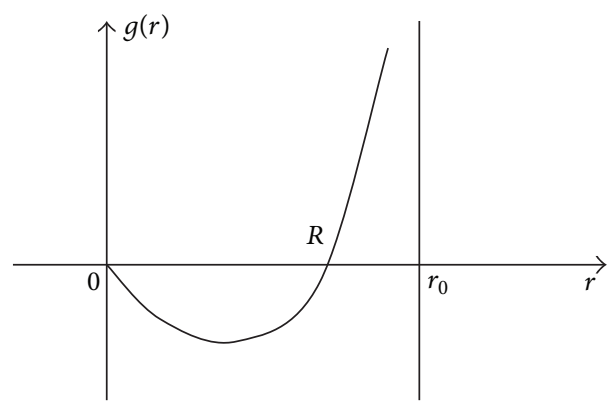

FIgUre 1: Adjustment coefficient $R$.

The following discussions are adjustment coefficient and the adjustment coefficient equation. Since the ruin probability as a number of indicators can evaluate insurance company solvency, it attracts attention. The research goal is to obtain specific expression of ruin probability. However, it is very difficult to directly obtain the expression of this function, but Lundberg found an indirect expression way by introducing a parameter which can play the intermediary role, namely, Lundberg coefficient or adjustment coefficient. Its principle is that the ruin probability is expressed as a function of adjustment coefficient and then seeks the calculation for adjustment coefficient. Thus, the adjustment coefficient plays a very important role in the study of ruin probability.

Lemma 5. Equation $g(r)=0$ is said to be an adjustment coefficient equation of the risk model (2), and it has a unique positive solution $r=R$, which is called an adjustment coefficient (see Figure 1).

Proof. We only need to prove that it has the following four properties:

(1) $g(0)=0$.

(2) $g^{\prime}(0)<0$.

(3) $g(r) \rightarrow+\infty(r \rightarrow+\infty)$.

(4) $g^{\prime \prime}(r)>0, \forall r \in(0,+\infty)$.

Obviously, $g(0)=0$.

Since

$$
\begin{aligned}
g^{\prime}(r)= & -A \alpha+\frac{\lambda(1-\rho) E[-X \exp (-r X)]}{\left(1-\rho M_{X}(-r)\right)^{2}} \\
& +\frac{\lambda p(1-\rho) E[Y \exp (r Y)]}{\left(1-\rho M_{Y}(r)\right)^{2}} \\
& +\frac{\lambda q(1-\rho) E[Z \exp (r Z)]}{\left(1-\rho M_{Z}(r)\right)^{2}}+\beta^{2} r
\end{aligned}
$$

and $M_{X}(0)=M_{Y}(0)=M_{Z}(0)=1$, then we have

$$
\begin{aligned}
g^{\prime}(0) & =-A \alpha-\frac{\lambda \mu_{X}}{1-\rho}+\frac{\lambda p \mu_{Y}}{1-\rho}+\frac{\lambda q \mu_{Z}}{1-\rho} \\
& =-\theta\left(\frac{\lambda p \mu_{Y}}{1-\rho}+\frac{\lambda q \mu_{Z}}{1-\rho}\right)<0 .
\end{aligned}
$$

Further,

$$
\begin{aligned}
g^{\prime \prime}(r) & =\frac{\lambda(1-\rho)\left(1-\rho M_{X}(-r)\right)}{\left(1-\rho M_{X}(-r)\right)^{4}} \\
& \cdot\left\{\left(1-\rho M_{X}(-r)\right) E\left[X^{2} e^{-r X}\right]\right. \\
+ & \left.2 \rho\left(E\left[-X e^{-r X}\right]\right)^{2}\right\}+\frac{\lambda \rho(1-\rho)\left(1-\rho M_{Y}(r)\right)}{\left(1-\rho M_{Y}(r)\right)^{4}} \\
& +\left\{\left(1-\rho M_{Y}(r)\right) E\left[Y^{2} e^{-r Y}\right]+2 \rho\left(E\left[Y e^{-r Y}\right]\right)^{2}\right\} \\
& +\frac{\lambda q(1-\rho)\left(1-\rho M_{Z}(r)\right)}{\left(1-\rho M_{Z}(r)\right)^{4}} \\
& +\left\{\left(1-\rho M_{Z}(r)\right) E\left[Z^{2} e^{-r Z}\right]+2 \rho\left(E\left[Z e^{-r Z}\right]\right)^{2}\right\} \\
& +\beta^{2} .
\end{aligned}
$$

It is easy to see the moment generating functions $M_{Y}(r)$, $M_{Z}(r)$ are increasing function, so there exists an $r_{1}>0$ such that $M_{Y}\left(r_{1}\right)=1 / \rho$ due to $0<\rho<1$. Similarly, there exists an $r_{2}>0$ such that $M_{Y}\left(r_{2}\right)=1 / \rho$. Let $r_{0}=\min \left\{r_{1}, r_{2}\right\}$; then, when $0<r<r_{0}$, we have $1<M_{Y}(r), M_{Z}(r),<1 / \rho$; that is, $1-\rho M_{Y}(r)>0$ and $1-\rho M_{Z}(r)>0$. When $r>0$, we have $M_{X}(-r)<1$; that is, $1-\rho M_{X}(-r)>0$. So $g^{\prime \prime}(r)>0(0<$ $\left.r<r_{0}\right)$ and $g(r)$ is a lower convex function. And because $\lim _{r \rightarrow+\infty} g(r)=+\infty$, then $g(r)=0$ has a unique positive solution $R$.

Theorem 6. The adjustment coefficient $R$ satisfies the following inequality:

$$
\begin{aligned}
& \frac{2\left[A \alpha+\lambda \mu_{X}\right]}{\lambda\left(\mu_{X}^{2}+\sigma_{X}^{2}\right)+\beta^{2}} \leq R \\
& \leq \frac{2\left[(1-\rho) A \alpha+\lambda \mu_{X}-\lambda p \mu_{Y}-\lambda q \mu_{Z}\right]}{\lambda\left[\left(\mu_{X}^{2}+\sigma_{X}^{2}\right)+p\left(\mu_{Y}^{2}+\sigma_{Y}^{2}\right)+q\left(\mu_{Z}^{2}+\sigma_{Z}^{2}\right)+\beta^{2}(1-\rho)\right]} .
\end{aligned}
$$

Proof. By Taylor's expansion, we have

$$
\begin{aligned}
M_{X}(-R) & =E\left[e^{-R X}\right]=E\left[1-R X+\frac{R^{2} X^{2}}{2}\right] \\
& =1-R \mu_{X}+\frac{R^{2}}{2}\left(\mu_{X}^{2}+\sigma_{X}^{2}\right) \\
M_{Y}(R) & =E\left[e^{R Y}\right]=E\left[1+R Y+\frac{R^{2} Y^{2}}{2}\right] \\
& =1+R \mu_{Y}+\frac{R^{2}}{2}\left(\mu_{Y}^{2}+\sigma_{Y}^{2}\right) .
\end{aligned}
$$


Then,

$$
\begin{aligned}
0= & g(R) \\
= & -R A \alpha+\frac{\lambda\left(M_{X}(-R)-1\right)}{1-\rho M_{X}(-R)}+\frac{\lambda p\left(M_{Y}(R)-1\right)}{1-\rho M_{Y}(R)} \\
& +\frac{\lambda q\left(M_{Z}(R)-1\right)}{1-\rho M_{Z}(R)}+\frac{1}{2} \beta^{2} R^{2} \\
\geq & -R A \alpha+\frac{\lambda\left[-R \mu_{X}+\left(R^{2} / 2\right)\left(\mu_{X}^{2}+\sigma_{X}^{2}\right)\right]}{1-\rho} \\
& +\frac{\lambda p\left[R \mu_{Y}+\left(R^{2} / 2\right)\left(\mu_{Y}^{2}+\sigma_{Y}^{2}\right)\right]}{1-\rho} \\
& +\frac{\lambda q\left[R \mu_{Z}+\left(R^{2} / 2\right)\left(\mu_{Z}^{2}+\sigma_{Z}^{2}\right)\right]}{1-\rho}+\frac{1}{2} \beta^{2} R^{2} .
\end{aligned}
$$

Dividing both sides of the above inequality by $R$, we obtain

$R$

$$
\leq \frac{2\left[(1-\rho) A \alpha+\lambda \mu_{X}-\lambda p \mu_{Y}-\lambda q \mu_{Z}\right]}{\lambda\left[\left(\mu_{X}^{2}+\sigma_{X}^{2}\right)+p\left(\mu_{Y}^{2}+\sigma_{Y}^{2}\right)+q\left(\mu_{Z}^{2}+\sigma_{Z}^{2}\right)+\beta^{2}(1-\rho)\right]} .
$$

Similarly,

$$
\begin{aligned}
0= & g(R) \\
\leq & -R A \alpha+\left[\lambda\left(M_{X}(-R)-1\right)\right]+\frac{\lambda p\left(M_{Y}(R)-1\right)}{1-M_{Y}(R)} \\
& +\frac{\lambda q\left(M_{Z}(R)-1\right)}{1-M_{Z}(R)}+\frac{1}{2} \beta^{2} R^{2} \\
\leq & -R A \alpha+\lambda\left[-R \mu_{X}+\frac{R^{2}}{2}\left(\mu_{X}^{2}+\sigma_{X}^{2}\right)\right]-\lambda p-\lambda q \\
& +\frac{1}{2} \beta^{2} R^{2} \\
\leq & -R A \alpha+\lambda\left[-R \mu_{X}+\frac{R^{2}}{2}\left(\mu_{X}^{2}+\sigma_{X}^{2}\right)\right]+\frac{1}{2} \beta^{2} R^{2} .
\end{aligned}
$$

Dividing both sides of the above inequality by $R$, we have

$$
R \geq \frac{2\left[A \alpha+\lambda \mu_{X}\right]}{\lambda\left(\mu_{X}^{2}+\sigma_{X}^{2}\right)+\beta^{2}} .
$$

For the profits process $\{S(t), t \geq 0\}$, let $F_{t}^{S}=\sigma(S(v), v \leq$ $t$ ) be a filtration. Let $T=\inf \{t: t \geq 0, U(t)<0\}$ and $\psi(u)=$ $\operatorname{Pr}\{T<\infty \mid U(0)=u\}$ be ruin time and ruin probability.

Lemma 7. $T$ is $F_{t}^{S}$-stopping time.

Theorem 8. $\left\{H_{u}(t), F_{t}, t \geq 0\right\}$ is a martingale, where $H_{u}(t)=$ $\exp [-r U(t)-\operatorname{tg}(r)]$.

Proof. In fact, $\{U(t), t \geq 0\}$ has stationary and independent increments, and from [2] we know that $H_{u}(t)$ is a martingale if and only if $E(\exp [-R U(t)])=\exp [-R u]$. By Lemmas 3 and 4 , there is a function $g(r)$ such that $E(\exp [-R S(t)])=$ $\exp [\operatorname{tg}(R)]=1$; then,

$$
\begin{aligned}
E(\exp [-R U(t)]) & =E[\exp [-R u]] E(\exp [-R S(t)]) \\
& =\exp [-R u],
\end{aligned}
$$

so $\left\{H_{u}(t), F_{t}, t \geq 0\right\}$ is a martingale.

Theorem 9. For any real number $r$, the ruin probability $\psi(u)$ satisfies

$$
\psi(u) \leq \exp (-r u) E\left[\sup _{t \geq 0} \exp (\operatorname{tg}(r))\right] .
$$

Proof. For a fixed time $t_{0}, t_{0} \wedge T$ is a bounded stopping time. Using the theorem of martingale and stopping time, we have

$$
\exp (-r u)=E\left[H_{u}(0)\right]=E\left[H_{u}\left(t_{0} \wedge T\right)\right] .
$$

By the full expectations formula, we have

$$
\begin{aligned}
\exp (-r u)= & E\left[H_{u}(T) \mid T \leq t_{0}\right] \operatorname{Pr}\left(T \leq t_{0}\right) \\
& +E\left[H_{u}\left(t_{0}\right) \mid T>t_{0}\right] \operatorname{Pr}\left(T>t_{0}\right) \\
\geq & E\left[H_{u}(T) \mid T \leq t_{0}\right] \operatorname{Pr}\left(T \leq t_{0}\right),
\end{aligned}
$$

which implies

$$
\begin{aligned}
\operatorname{Pr}\left(T \leq t_{0}\right) & =\frac{\exp (-r u)}{E\left[H_{u}(T) \mid T \leq t_{0}\right]} \\
& \leq \frac{\exp (-r u)}{\inf _{0 \leq t \leq t_{0}}[\exp (-\operatorname{tg}(r))]} \\
& =\exp (-r u) \cdot \sup _{0 \leq t \leq t_{0}}[\exp (\operatorname{tg}(r))] .
\end{aligned}
$$

By expectation on both sides of the above inequality and letting $t_{0} \rightarrow \infty$, we can get the desired results.

Theorem 10. The ruin probability of surplus process $\{U(t) ; t \geq$ $0\}$ satisfies

$$
\psi(u)=\frac{\exp (-R u)}{E[\exp (-R U(T)) \mid T<\infty]} .
$$

Proof. For a fixed time $t_{0}, t_{0} \wedge T$ is a bounded stopping time. Using the theorem of martingale and stopping time, we have

$$
\begin{aligned}
\exp (-r u)= & E\left[H_{u}\left(t_{0} \wedge T\right)\right] \\
= & E\left[H_{u}(T) \mid T \leq t_{0}\right] \operatorname{Pr}\left(T \leq t_{0}\right) \\
& +E\left[H_{u}\left(t_{0}\right) \mid T>t_{0}\right] \operatorname{Pr}\left(T>t_{0}\right) .
\end{aligned}
$$

Let $r=R$, we have

$$
\begin{aligned}
\exp (-R u) & \\
= & E\left[\exp (-R U(T)) \mid T \leq t_{0}\right] \operatorname{Pr}\left(T \leq t_{0}\right) \\
& +E\left[\exp (-R U(T)) \mid T>t_{0}\right] \operatorname{Pr}\left(T>t_{0}\right),
\end{aligned}
$$


which implies

$$
\begin{aligned}
0 & \leq E\left[\exp (-R U(T)) \mid T>t_{0}\right] \operatorname{Pr}\left(T>t_{0}\right) \\
& \leq E\left[\exp \left(-R U\left(t_{0}\right)\right) I\left(U\left(t_{0}\right) \geq 0\right)\right] .
\end{aligned}
$$

Since $0 \leq \exp \left(-R U\left(t_{0}\right)\right) I\left(U\left(t_{0}\right) \geq 0\right) \leq 1$, by the law of large numbers, when $t_{0} \rightarrow \infty, U\left(t_{0}\right) \rightarrow \infty$ (a.s.). By dominated convergence theorem, we have

$$
\lim _{t_{0} \rightarrow \infty} E\left[\exp (-R U(T)) \mid T>t_{0}\right] \operatorname{Pr}\left(T>t_{0}\right)=0,
$$

(a.s.) .

Then, when $t_{0} \rightarrow \infty$ in (26), we can obtain (22).

Corollary 11. For the surplus process $\{U(t) ; t \geq 0\}$, the ruin probability $\psi(u)$ satisfies Lundberg inequality:

$$
\psi(u) \leq \exp (-R u), \quad u \geq 0,
$$

where $R$ is adjustment coefficient.

In order to get this inequality as good as possible, we shall choose $r$ as large as possible under the restriction $\sup _{t \geq 0} \exp (\operatorname{tg}(r))<\infty$. Combined with Figure 1, we have $R=\sup \{r \mid g(r) \leq 0\}$.

Theorem 12. The ruin probability of insurance company before timet satisfies

$$
\psi_{t}(u) \leq \begin{cases}e^{-R_{y} u}, & t<\frac{u}{g^{\prime}(R)} \\ e^{-R u}, & t \geq \frac{u}{g^{\prime}(R)},\end{cases}
$$

where $R_{y}=f\left(r_{y}\right)$ and $r_{y}$ is the solution of $g^{\prime}(r)=u / t$.

Proof. By Lemma 4, we have

$$
\begin{aligned}
\psi_{t}(u) & \leq e^{-r u} \cdot \sup _{0 \leq t \leq \infty}\left[e^{t g(r)}\right]=e^{-r u} \cdot \max \left\{1, e^{t g(r)}\right\} \\
& \leq e^{-u \min \{r, r-(t / u) g(r)\}}=e^{-u \min \{r, f(r)\}},
\end{aligned}
$$

where $f(r)=r-y g(r), y=t / u$. Obviously, the supremum of $\psi_{t}(u)$ is $e^{-u \sup _{r \geq 0}\{\min \{r, f(r)\}\}}$.

Since $f(r)$ is the convex function and $f(R)=R$, when $r>R$, we have $f(r)<r$; when $0<r<R$, we have $f(r)>r$. Let $r_{y}$ be the solution of $f^{\prime}(r)=0$ and $R_{y}=f\left(r_{y}\right)$; then, $R_{y}$ is the maximum value of $f(r)$.

And because

$$
\begin{aligned}
& r_{y} \leq R, \quad t>\frac{u}{g^{\prime}(R)} \\
& r_{y}>R, \quad t \leq \frac{u}{g^{\prime}(R)}
\end{aligned}
$$

then, we have

$$
\sup _{r \geq 0}\{\min [r, f(r)]\}= \begin{cases}R_{y}, & t<\frac{u}{g^{\prime}(R)} \\ R, & t \geq \frac{u}{g^{\prime}(R)} .\end{cases}
$$

Theorem 13. Let $\tau=\inf \{t \geq 0, U(t)=x>u\}$ be the time when the surplus reaches a given level firstly; then, the Laplace transform of $\tau$ is as follows:

$$
E\left[e^{-s \tau}\right]=e^{r x}
$$

where $s$ and $r$ satisfy $s=g(r)$.

Proof. For the surplus process $\{U(t) ; t \geq 0\}$, using the theorem of martingale and stopping time, we see that $\tau$ is a stopping rime of $F_{t}^{S}$. Let $H(t)=e^{-r U(\tau)-s \tau}$. By Theorem 8, the surplus process $\{H(t) ; t \geq 0\}$ is a martingale. Hence, we have $E[H(\tau)]=E[H(0)]$; that is, $E\left[e^{-r U(\tau)-s \tau}\right]=1$. Since $U(\tau)=x$, then we get $E\left[e^{-r x-s \tau}\right]=1$; that is, $E\left[e^{-s \tau}\right]=e^{r x}$.

Theorem 14. The expectation and variance of $\tau$ satisfy

$$
\begin{aligned}
E[\tau] & =\frac{x}{\eta} \\
\operatorname{Var}[\tau] & =\frac{x \omega}{\eta^{3}},
\end{aligned}
$$

where

$$
\begin{aligned}
\eta= & A \alpha+\frac{\lambda \mu_{X}}{1-\rho}-\frac{\lambda p \mu_{Y}}{1-\rho}-\frac{\lambda q \mu_{Z}}{1-\rho} \\
\omega= & \frac{\lambda}{(1-\rho)^{2}}\left\{(1-\rho)\left(\sigma_{X}^{2}+\mu_{X}^{2}\right)+2 \rho \mu_{X}^{2}\right\} \\
& +\frac{\lambda p}{(1-\rho)^{2}}\left\{(1-\rho)\left(\sigma_{Y}^{2}+\mu_{Y}^{2}\right)+2 \rho \mu_{Y}^{2}\right\} \\
& +\frac{\lambda q}{(1-\rho)^{2}}\left\{(1-\rho)\left(\sigma_{Z}^{2}+\mu_{Z}^{2}\right)+2 \rho \mu_{Z}^{2}\right\}+\beta^{2}
\end{aligned}
$$

Proof. By Theorem 13, we have $E\left[e^{-s \tau}\right]=e^{r x}$; let $\varphi(s)=$ $\ln E\left[e^{-s \tau}\right]$; that is, $\varphi(s)=r x$; then, we have the following:

$$
\begin{aligned}
& \varphi^{\prime}(s)=\frac{d \varphi(s)}{d r} \cdot \frac{1}{d s / d r}=\frac{x}{s^{\prime}(r)}=\frac{x}{g^{\prime}(r)} \\
& \varphi^{\prime \prime}(s)=\frac{d \varphi^{\prime}(s)}{d s}=\frac{d \varphi^{\prime}(s)}{d r} \cdot \frac{1}{s^{\prime}(r)}=-\frac{x g^{\prime \prime}(r)}{\left[g^{\prime}(r)\right]^{3}} .
\end{aligned}
$$

By Lemma 5, we have

$$
\begin{aligned}
& E[\tau]=-\left.\frac{d \varphi(s)}{d s}\right|_{s=r=0}=-\frac{x}{g^{\prime}(0)} \\
& =-\frac{x}{-A \alpha-\lambda \mu_{X} /(1-\rho)+\lambda p \mu_{Y} /(1-\rho)+\lambda q \mu_{Z} /(1-\rho)} \\
& =\frac{x}{\eta} \\
& \operatorname{Var}[\tau]=\left.\varphi^{\prime \prime}(s)\right|_{s=r=0}=\frac{x \omega}{\eta^{3}} .
\end{aligned}
$$

Thus, Theorem 12 is obtained. 
TABLE 1: The upper bound of the ruin probability.

\begin{tabular}{lcccccccccccc}
\hline $1 / b$ & \multirow{2}{*}{$R$} & \multicolumn{1}{c}{$\operatorname{cxp}(-R u)$} \\
& & $u=1000$ & $u=1100$ & $u=1200$ & $u=1300$ & $u=1400$ & $u=1500$ & $u=1600$ & $u=1700$ & $u=1800$ & $u=2000$ & $u=10000$ \\
\hline $1 / 1000$ & 0.0010 & 0.3677 & 0.3326 & 0.3010 & 0.2723 & 0.2464 & 0.2229 & 0.2017 & 0.1825 & 0.1651 & 0.1325 & 0.00004 \\
$1 / 1100$ & $9.0960 e-004$ & 0.4027 & 0.3677 & 0.3357 & 0.3065 & 0.2799 & 0.2555 & 0.2333 & 0.2130 & 0.1945 & 0.1622 & 0.00011 \\
$1 / 1200$ & $8.3376 e-004$ & 0.4344 & 0.3997 & 0.3677 & 0.3383 & 0.3112 & 0.2863 & 0.2634 & 0.2423 & 0.2230 & 0.1887 & 0.00024 \\
$1 / 1300$ & $7.6959 e-004$ & 0.4632 & 0.4289 & 0.3971 & 0.3677 & 0.3405 & 0.3152 & 0.2919 & 0.2703 & 0.2503 & 0.2146 & 0.00045 \\
$1 / 1400$ & $7.1460 e-004$ & 0.4894 & 0.4556 & 0.4242 & 0.3950 & 0.3677 & 0.3424 & 0.3187 & 0.2968 & 0.2763 & 0.2395 & 0.00079 \\
$1 / 1500$ & $6.6694 e-004$ & 0.5133 & 0.4802 & 0.4492 & 0.4202 & 0.3931 & 0.3667 & 0.3440 & 0.3218 & 0.3010 & 0.2635 & 0.0013 \\
$1 / 1600$ & $6.2524 e-004$ & 0.5351 & 0.5027 & 0.4722 & 0.4436 & 0.4167 & 0.3915 & 0.3677 & 0.3455 & 0.3245 & 0.2864 & 0.0019 \\
$1 / 1700$ & $5.8845 e-004$ & 0.5552 & 0.5235 & 0.4935 & 0.4653 & 0.4387 & 0.4137 & 0.3900 & 0.3667 & 0.3467 & 0.3082 & 0.0028 \\
$1 / 1800$ & $5.5574 e-004$ & 0.5736 & 0.5426 & 0.5133 & 0.4856 & 0.4953 & 0.4345 & 0.4110 & 0.3888 & 0.3678 & 0.3291 & 0.0039 \\
$1 / 1900$ & $5.2649 e-004$ & 0.5907 & 0.5604 & 0.5316 & 0.5044 & 0.4785 & 0.4540 & 0.4307 & 0.4086 & 0.3876 & 0.3489 & 0.0052 \\
$1 / 200055.0015 e-004$ & 0.6064 & 0.5769 & 0.5487 & 0.5219 & 0.4965 & 0.4723 & 0.4492 & 04273 & 0.4065 & 0.3678 & 0.0067 \\
\hline
\end{tabular}

\section{Numerical Simulation and Analysis}

4.1. Simulation of the Upper Bound of Ruin Probability. Since the adjustment coefficient $R$ can be used to measure the risk, by formula $\psi(u) \leq \exp (-R u)$, we know that the higher adjustment coefficient $R$ results in the less ruin probability. The following will be used to simulate the size of ruin probability by adjustment coefficient $R$.

Suppose $\lambda=40, \rho=0.1, A=400, \alpha=0.1, \beta=1$, $p=0.005$, and $q=0.0005$. Random variables $X_{k}, Y_{k}$, and $Z_{k}$ obey the exponential distribution with mean $\mu_{X}=b, \mu_{Y}=20$, and $\mu_{Z}=10$, respectively. Taking different values about $b$ and $u$, by MATLAB and Theorem 6 , we can get the upper bound of the ruin probability. See Table 1.

Table 1 shows that ruin probability of insurance company varies tremendously in size with different value of $u$; the higher initial surplus $u$ results in the less ruin probability. Magnitude of initial capital increase is far below the level of reduced number of ruin probability of insurance company. For example, in the first seven behaviors of Table 1, the initial capital $u$ is only increased by 10 times, and the ruin probability decreases from 0.4894 to 0.00079 . This is another example in the course of business; the availability of sufficient initial capital is crucial to the insurance company.

In addition, determining the value of distribution parameter $1 / b$ of the premium $X$ has a great impact on the ruin probability of insurance company. For the exponential distribution, the value of $1 / b$ is smaller; the smaller the amount of premium charged by insurance company, the greater the probability of ruin. This suggests that a reasonable determination of the premium on the normal operation of insurance companies is very important, which leads to higher requirement for determination of the premium in the design of insurance products in the insurance company.

4.2. Simulation of the First Arrival Time. Suppose $\lambda=40$, $\rho=0.1, A=5000, \alpha=0.1, x=2000, \beta=1, p=0.005$, and $q=0.0005$ (in order to clear the trend of curve, in Figure 4, let $q=0.0125$; in Figure 8 , let $q=0.005$ ). Random variables $X_{k}, Y_{k}$, and $Z_{k}$ obey the exponential distribution with mean $\mu_{X}=100, \mu_{Y}=80$, and $\mu_{Z}=60$, respectively. We obtained the trend chart of average time and the variance of time.

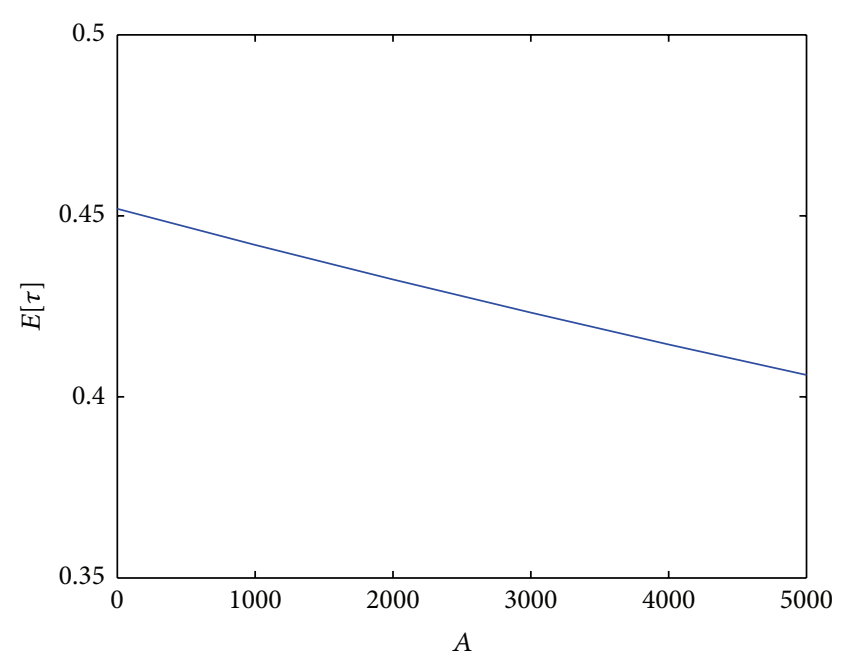

Figure 2

(1) The Trend Figure of Average Time. From Figures 2-5, we can clearly know that the first arrival time to reach a given level is a decreasing function of investment capital $A$ and investment interest rate $\alpha$ and is an increasing function of claim rate $\lambda p$ and surrender rate $\lambda q$.

In comparison, the change in the average time is more sensitive to the change of investment capital and investment rates and is not sensitive to the change of claim rate and surrender rate.

(2) The Trend Figure of Variance of the First Arrival Time. Figures 6-9 point out that the first arrival time to reach a given level is a decreasing function of investment capital $A$ and investment interest rate $\alpha$ and is an increasing function of claim rate $\lambda p$ and surrender rate $\lambda q$. In comparison, the change in variance is more sensitive to the change of investment capital and investment rates and is not sensitive to the change of claim rate and surrender rate. 


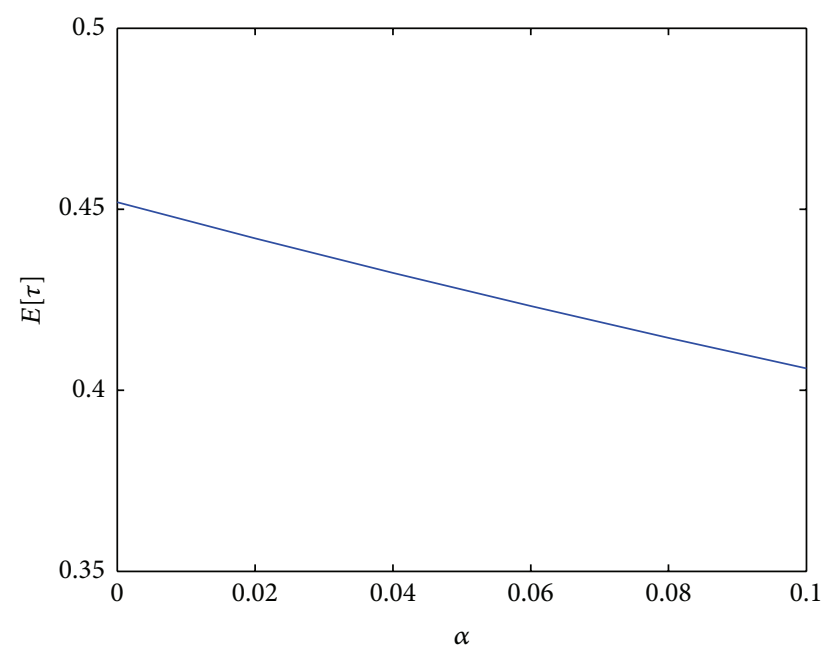

FIgURe 3

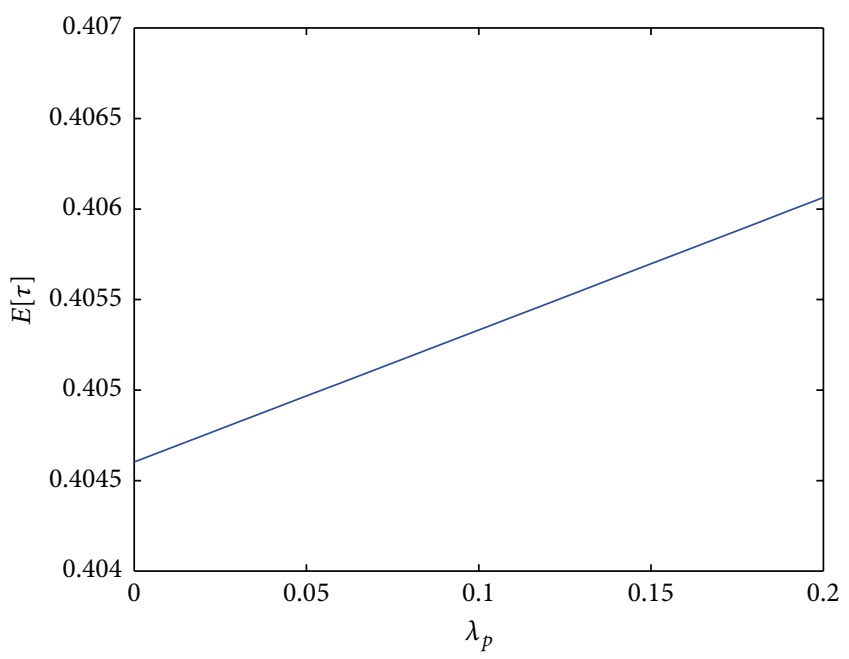

FIGURE 4

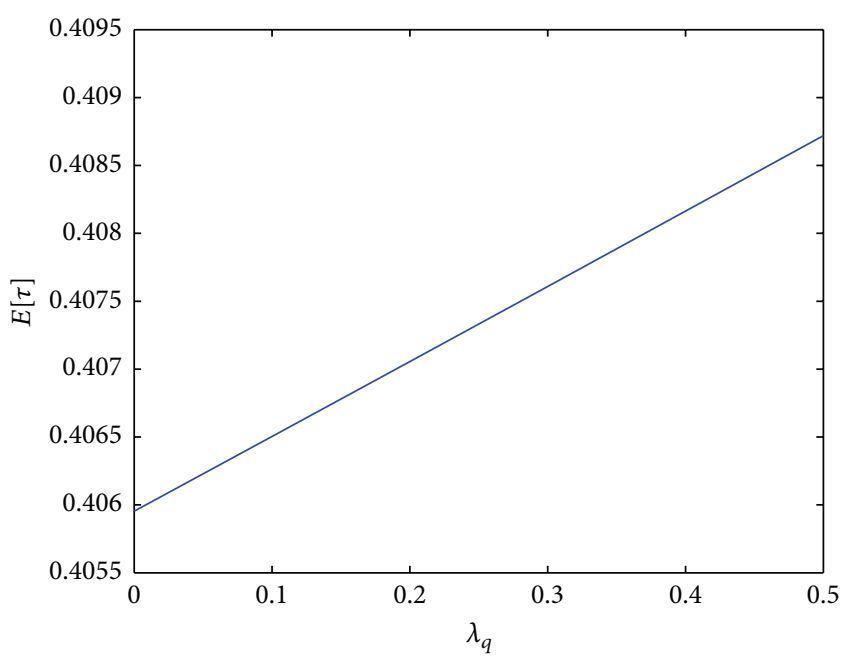

FIGURE 5

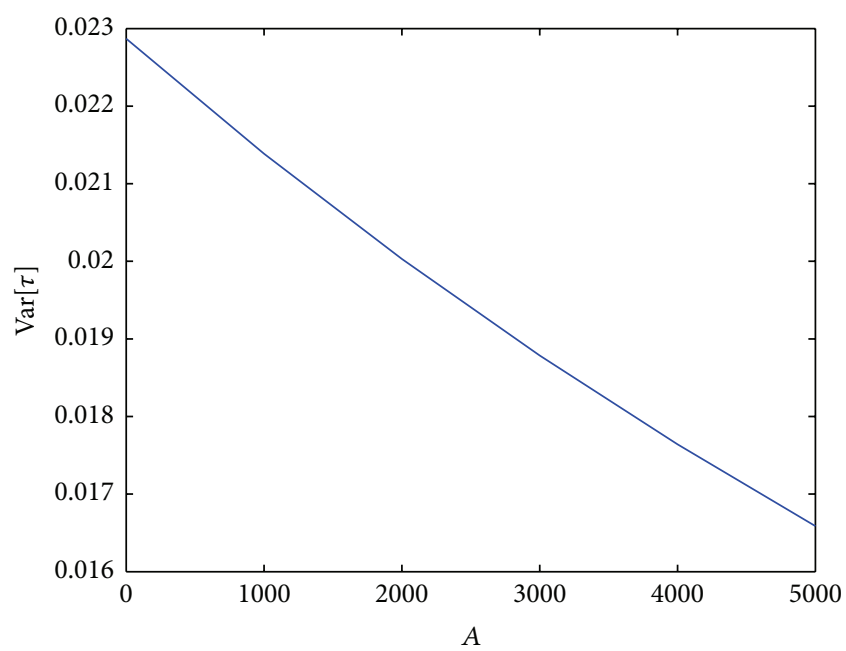

Figure 6

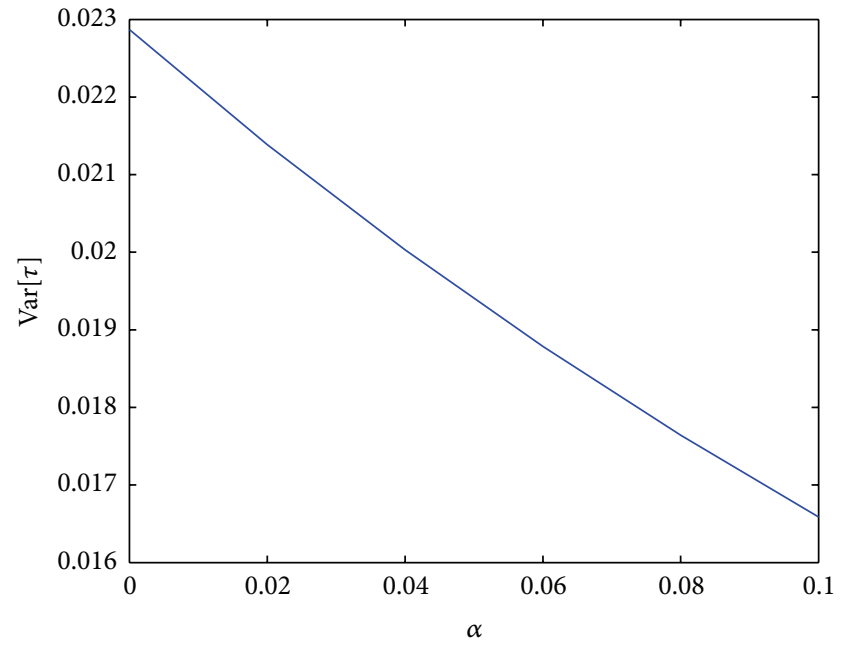

FIGURE 7

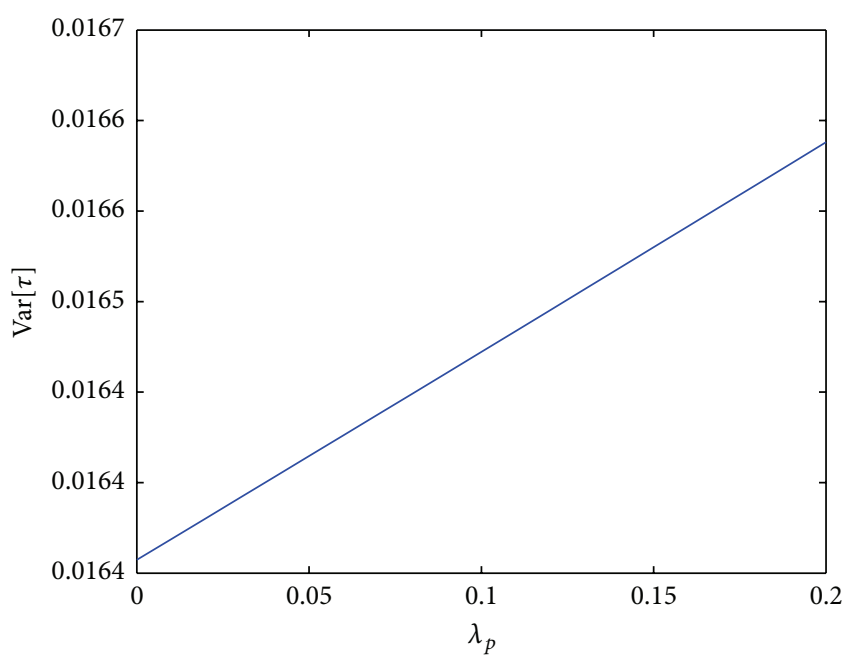

FIGURE 8 


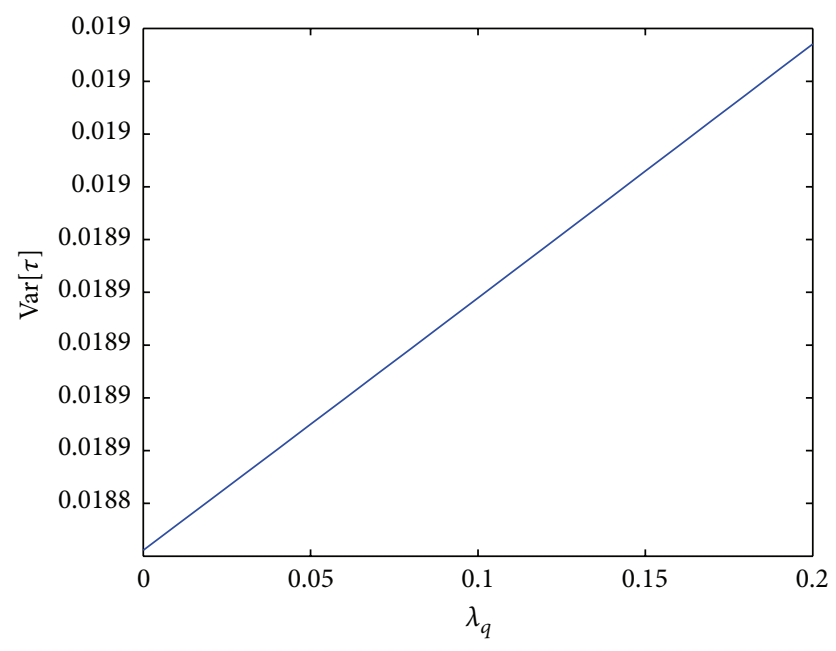

Figure 9

\section{Conflict of Interests}

The authors declare that there is no conflict of interests regarding the publication of this paper.

\section{Acknowledgments}

This work was supported by National Social Science Foundation of China (no. 15BJY007), National Natural Science Foundation of China (no. 11301303 and no. 11231005), Humanities and Social Sciences Project of the Ministry of Education of China (no. 14YJA630088, no. 14YJC790054, no. 13YJA790035, no. 13YJC630150, and no. 13YJC630162), Natural Science Foundation of Shandong Province (no. ZR2012AQ013 and no. ZR2010GL013), A Project of Shandong Province Higher Educational Science and Technology Program (J15LI03, J15LI53), the 2014 Youth Talent Support Program of Shandong University of Finance and Economics, and the Startup Foundation of Doctor Scientific Research of Shandong Jiaotong University.

\section{References}

[1] H. U. Gerber, An Introduction to Mathematical Risk Theory, vol. 8 of S. S. Heubner Foundation Monograph Series, 1979.

[2] H. U. Gerber and E. S. W. Shiu, "On the time value of ruin," North American Actuarial Journal, vol. 2, no. 1, pp. 48-78, 1998.

[3] J. Liu, J. C. Xu, and Y. J. Hu, “On the expected discounted penalty function in a Markov-dependent risk model with constant dividend barrier," Acta Mathematica Scientia B, vol. 30, no. 5, pp. 1481-1491, 2010.

[4] P. Shi, X. P. Feng, and A. Ivantsova, "Dependent frequencyseverity modeling of insurance claims," Insurance: Mathematics \& Economics, vol. 64, pp. 417-428, 2015.

[5] T. Jiang, Y. Wang, Y. Chen, and H. Xu, "Uniform asymptotic estimate for finite-time ruin probabilities of a time-dependent bidimensional renewal model," Insurance: Mathematics and Economics, vol. 64, pp. 45-53, 2015.

[6] Z. M. Zhang and H. Yang, "Gerber-Shiu analysis in a perturbed risk model with dependence between claim sizes and interclaim times," Journal of Computational and Applied Mathematics, vol. 235, no. 5, pp. 1189-1204, 2011.

[7] Y. F. Shi, P. Liu, and C. S. Zhang, "On the compound Poisson risk model with dependence and a threshold dividend strategy," Statistics \& Probability Letters, vol. 83, no. 9, pp. 1998-2006, 2013.

[8] W. Zou, J.-W. Gao, and J.-H. Xie, "On the expected discounted penalty function and optimal dividend strategy for a risk model with random incomes and interclaim-dependent claim sizes," Journal of Computational and Applied Mathematics, vol. 255, pp. 270-281, 2014.

[9] Z. C. Mao and J. E. Liu, "The distribution about numbers of claims on homogeneous policyholders under NCD system and stop loss insurance," Chinese Journal of Management Science, vol. 13, no. 5, pp. 1-5, 2005.

[10] W. Yu, "Some results on absolute ruin in the perturbed insurance risk model with investment and debit interests," Economic Modelling, vol. 31, no. 1, pp. 625-634, 2013.

[11] G. H. Guan and Z. X. Liang, "Optimal reinsurance and investment strategies for insurer under interest rate and inflation risks," Insurance: Mathematics and Economics, vol. 55, pp. 105115, 2014.

[12] W. Yu, "Randomized dividends in a discrete insurance risk model with stochastic premium income," Mathematical Problems in Engineering, vol. 2013, Article ID 579534, 9 pages, 2013.

[13] Y. J. Huang and W. G. Yu, "Studies on a double poissongeometric insurance risk model with interference," Discrete Dynamics in Nature and Society, vol. 2013, Article ID 128796, 8 pages, 2013.

[14] Z. C. Mao and J. E. Liu, "The expression of ruin probability under claim number with compound Poisson-Geometric process," Chinese Journal of Management Science, vol. 15, no. 5, pp. 23-28, 2007.

[15] X. Lin and N. Li, "Ruin probability, optimal investment and reinsurance strategy for an insurer with compound poissongeometric risk process," Mathematica Applicata, vol. 24, no. 1, pp. 174-180, 2011. 


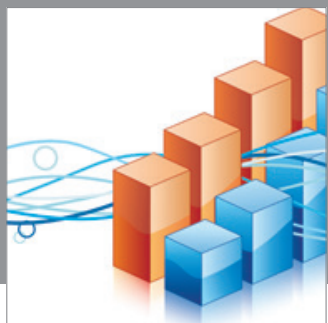

Advances in

Operations Research

mansans

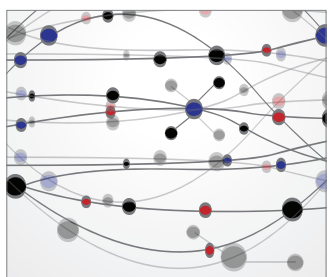

The Scientific World Journal
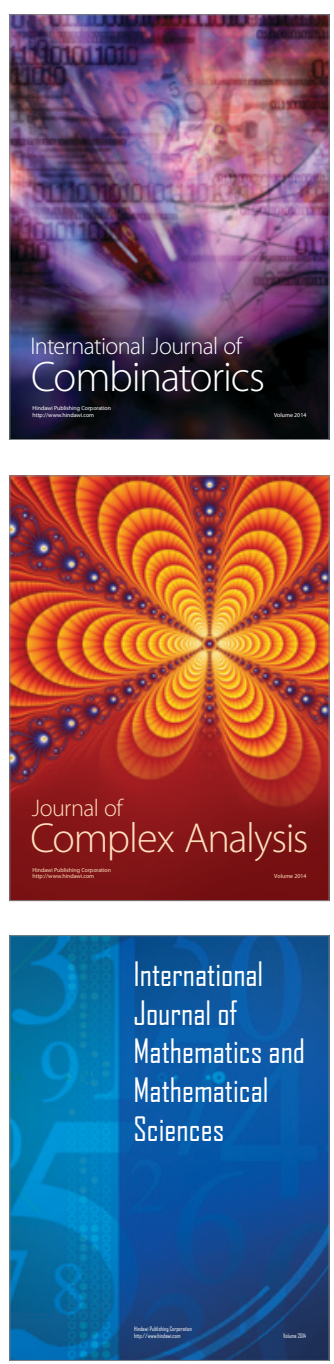
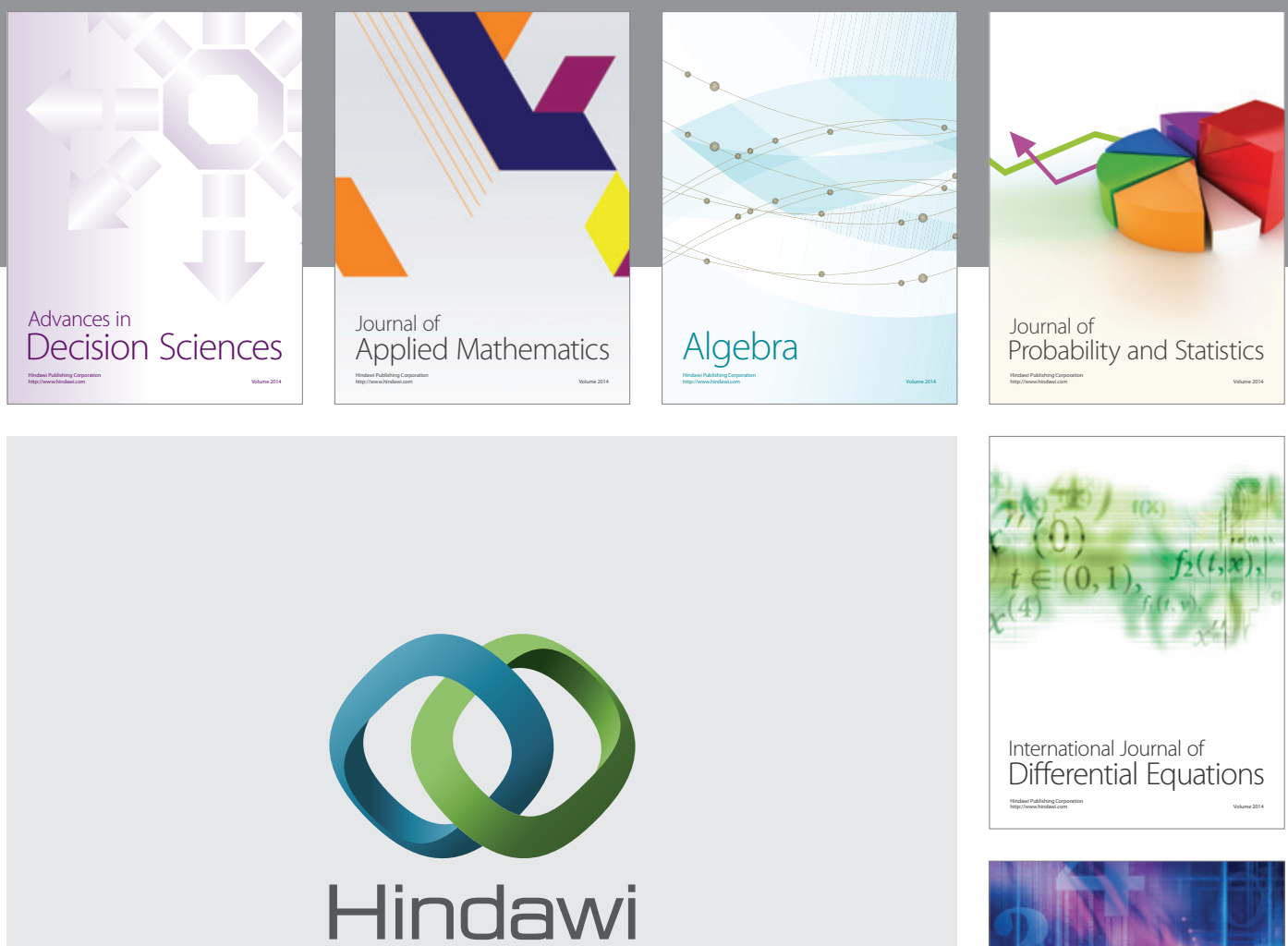

Submit your manuscripts at http://www.hindawi.com
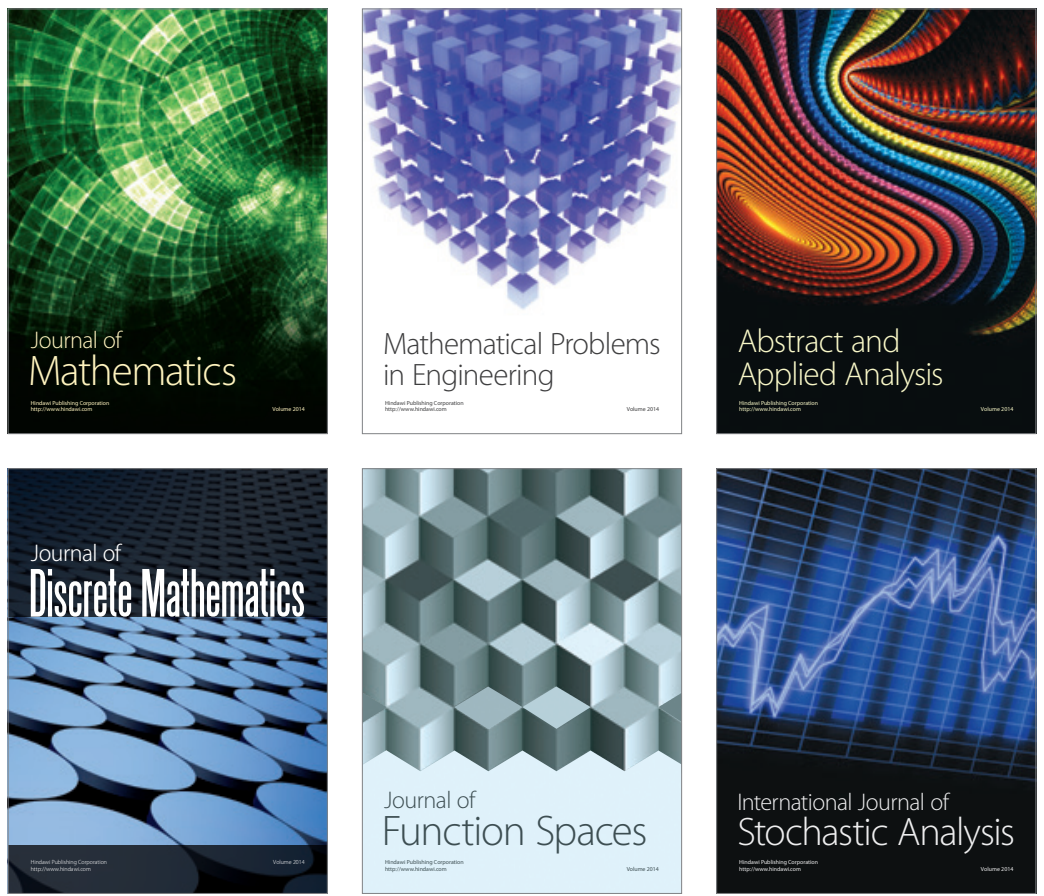

Journal of

Function Spaces

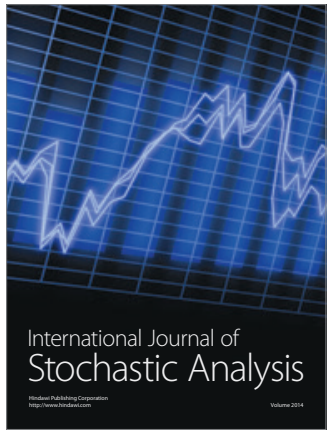


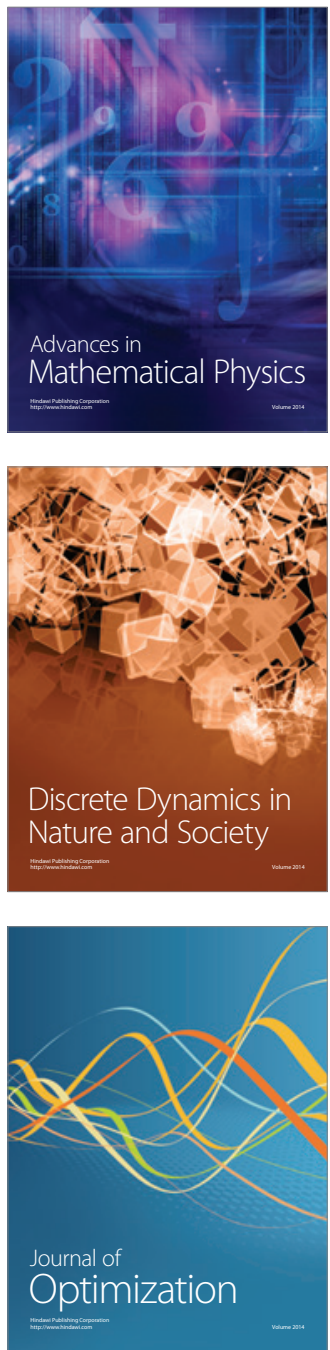